\title{
Validation of a Digital Pathology Whole Slide Imaging System
}

\author{
B.E. Maleeff \\ Department of Safety Assessment, GlaxoSmithKline, King of Prussia, PA
}

Digital whole slide imaging (WSI) provides a complement to traditional histopathology assessments of glass tissue slides for toxicologic pathology. WSI allows users to view images of entire slide content, or selected fields at increasing magnifications, and to compare multiple slide images simultaneously. The technology incorporates robust image databases with metadata and search \& retrieval capabilities, and enables the use of digital slide images for cross-site collaboration and teleconsultation.

In previous presentations the selection, development and implementation of a non-Good Laboratory Practice (GLP)-compliant digital pathology system were discussed [1,2]. However, the content of the system could be used only for consultative or illustrative purposes and not for diagnosis or submission of preclinical regulatory studies. This presentation will describe the validation of a digital pathology WSI system for use in a regulated nonclinical environment to meet predicate rule and electronic records requirements (21CFR58, 21CFR11) [3].

The WSI system consists of: multiple site-based digital slide scanners; proprietary relational image database with central application server, database server, multiple site-based image file repositories and image servers; client-side freeware slide image viewer; image analysis algorithms; and a server-side real-time collaboration tool. In addition, a secure firewall for internal data security and external-facing firewall security complete the system design.

The validation team members, representing various disciplines in information technology, GLP \& computer systems quality assurance, and Safety Assessment scientific, risk management and computer systems validation personnel performed installation qualification (IQ) and operational qualification (OQ) testing as part of system implementation. In order for the system to pass performance qualification (PQ) so that it could be considered fit-for-purpose, both hardware and software components were tested by trained users with increasing levels of system access. Tester roles represented included a super user, system archivist, slide scanner operator, in-house pathologist client, and external pathologist client.

Points considered when testing scripts were authored included internal and external data security; data transmittal over networks; image quality and integrity; digital data management including data storage, back-up and retrieval; risk mitigation; audit trail integrity; data archival; and records retention. Standard operating procedures covering these topics were published as the penultimate step in the validation process.

The result of the project is a fit-for-purpose, GLP-compliant digital pathology WSI system. Although the US Food and Drug Administration has not yet approved this technology as a toxicologic pathology diagnostic platform, we anticipate that efforts such as this, and forthcoming proof-of-concept studies, will demonstrate the robustness of this application. 


\section{References:}

[1] B.E.Maleeff and J.R.Ormand, Microscopy and Microanalysis 16 (Suppl.2): 652-653, 2010.

[2] B.E.Maleeff, Microscopy and Microanalysis 17 (Suppl.2): 1124-1125, 2011.

[3] Code of Federal Regulations, United States Government.

[4] The author acknowledges the valuable contributions and collaboration of the validation project team members. 